\title{
Rational Asset Pricing Implications from Realistic Trading Frictions
}

\author{
by \\ Jean-Pierre Zigrand* \\ Department of Accounting and Finance, \\ and Financial Markets Group \\ The London School of Economics \\ Houghton Street, London WC2A $2 A E$ \\ E-mail: j.p.zigrand@lse.ac.uk
}

March 21, 2002

*I thank Pierre-André Chiappori, Roger Guesnerie, Erzo Luttmer, Joseph Perktold, Heracles Polemarchakis, José Scheinkman and participants at the CEPR Summer Symposium in Gerzensee, the Workshop on Markets and Information in Venice, the University of Amsterdam, Brunel University, CORE/Université Catholique de Louvain, the London School of Economics and the University of Essex for helpful comments. All errors are mine. 


\begin{abstract}
We study a very simple rational expectations (RE) model whose asset pricing implications address some of the short-run mispricings, informational inefficiencies and overreactions observed in real markets, without a need to resort to behavioural assumptions. We accomplish this by relying on the plausible joint frictions of immediacy risk (execution risk) and of asset-specific orders (the demand function for asset $a$ cannot be made contingent on the price of any asset other than $a$ ). These frictions induce allocational and informational inefficiencies akin to the ones observed in reality. At the closed-form RE Equilibrium it is shown that arbitrage opportunities occur which could not have occurred in a standard model. A certain and precise degree of informativeness of prices to the traders is lost because the decision making process becomes endogenously segmented and decentralized within the same decision making entity (distinct "trading desks"). It is shown that, compared to the frictionless benchmark case, volatility is affected at a RE Equilibrium, and that asset prices are likely to overreact to news. Interestingly, the coordination problem arising from limited communication, even though dramatically changing demand functions, does not lead to welfare losses.
\end{abstract}

JEL Classification: G11, G12, G14 


\section{Introduction}

Until recently most theoretical research in financial economics has been based on perfect, efficient and frictionless markets. But the pricing predictions thus derived are at best approximations to real world prices. Prices occasionally stray from their fundamental values (or from the values of a replicating portfolio), they may momentarily allow for arbitrage opportunities, sometimes they overreact to contemporaneous news, they may be either overly or insufficiently volatile, they fail to be informationally efficient, and the list goes on. The recent literature on behavioral finance provides some answers to these pricing patterns, as does the literature incorporating frictions such as transaction costs, short-sale constraints and tax considerations into rational general equilibrium models. This paper belongs to the frictional economics literature. Focusing on contemporaneous valuation errors, we propose a simple two-period model that generates rational competitive equilibrium asset prices which nevertheless exhibit many of the puzzling properties listed above. Prices of different assets are shown to allow for arbitrage opportunities with positive probability, information is not properly aggregated into prices, agents behaviour appears at odds with Bayesian updating, and we argue that overreactions are a natural outcome.

The framework we propose drops two simplifying (but arguably counterfactual) assumptions about the securities trading environment regularly made in financial economics. The first common assumption is that investors or traders know the price at which their orders will be executed. This is at best an approximation, valid possibly for orders of small sizes. Typically, traders face immediacy risk or trading lags (and if using strict limit orders the size of the execution remains random). The second assumption we drop in this paper concerns the kind of orders that can be submitted. Typically, investors are assumed to submit their Marshallian demand schedules to the market, either to an auction or to a market maker. Real world trading systems, however, do not allow orders for asset $a$ to be contingent upon the prices of any other assets $b \neq a$. In other words, schedules need to be asset specific. These two realistic trading frictions are shown to be sufficient to introduce mispricings in the form of allocational inefficiencies and arbitrage opportunities. In this paper it is also shown that informational inefficiencies arise at equilibrium, as the joint information of an investor effectively be-

comes compartmented into distinct information sets that can be thought of as belonging to partially informed "trading desks." Information processing 
and trading becomes decentralized and uncoordinated, albeit synergetic.

We have two interpretations of the microstructure described above in mind. The literal interpretation focuses on the very short-term. In realtime, the proposed trading environment does indeed resemble very much the real-world environment. An investor who wants to rebalance his portfolio does typically not know for sure the execution prices of his trades beforehand because of new information that gets impounded into prices between the moment the orders are submitted and the moment the price is confirmed. This is reminiscent of the timing in Kyle(1985)), but there investors may only submit market orders for the one asset that is traded. One way to internalize that execution uncertainty is to submit Marshallian demand schedules that have all contingencies across all assets incorporated. But that is exactly what is not feasible in the real world. It is shown that the resulting equilibrium asset prices may look irrational, or admit slight mispricings or fail to aggregate all the information that they would if markets were perfect. We are thus contributing both to the literature on market frictions that generate mispricings, and to the literature on the limits of arbitrage that studies why maximizing agents do not eliminate such mispricings. For papers on the latter, with an emphasis on its intertemporal long term aspects, see for instance De Long, Shleifer, Summers and Waldmann(1990)), Dow and Gorton(1994)) or Shleifer and Vishny(1997)). In contrast, given the assumption of two periods only, the model we propose may best describe short term mispricings, rather than long term valuation errors such as the closed-end funds puzzle, and its predictions may be complementary to alternative explanations of long-term inefficiencies.

A less literal interpretation may be to view immediacy risk not so much as execution risk, but more as arising from limited communication and decentralized decision making within the same decision-making firm. It is an uncontentious fact that not all decision makers within the same financial institution share all information, so that in effect their individual information sets do not coincide with the join, the coarsest common refinement. Either due to regulatory restrictions (e.g. "Chinese Wall") or due to practical and technical difficulties, various trading desks operate more or less independently in real time (with the possible exception of a firm-wide riskmanagement team). That does not, however, imply that the various traders disregard the actions of the other traders within the same firm. This is particularly true when the compensation of the traders is not only a function of individual or desk P\&L but of the $\mathrm{P} \& \mathrm{~L}$ of a division or a firm, as assumed 
here. More realistically, different traders roughly know the trades and strategies that need to be executed by the other traders, but are reduced to rely on second-guesses as to what their exact trades are at each moment. Assuming that each trader exactly knows all trades and that they all share the same information set is clearly unrealistic, and it is the role of this perfect information assumption that we try to address in this paper. In that spirit, we may extend the literal interpretation of our model and consider it applicable to mispricing patterns that may endure for some time in the markets since an active communication effort on the part of the various trading desks is required in order to sit together, to discover profitable trading opportunities and to jointly act upon them in a coordinated fashion.

More formally then, the desire to derive a closed-form solution necessitates strong, and possibly irrealistic, assumptions. In particular, we resort to quadratic utility functions. The model assumes price-taking investors who submit their asset demand schedules to an auction that computes aggregate demand schedules and clears markets. But due to the trading frictions, the decision problem of the investor is slightly more elaborate than usually. If the investor has rational expectations, then he tries to partially overcome the frictions by refining his information by the information revealed by prices as follows: even though the orders cannot be revised instantaneously after they're submitted, at the outset the investor built in as many contingencies as possible into the demand schedules (one for each asset by market specificity).

This means that we can view investor $h$ 's optimization program as a decentralized team maximization problem with as many team members (traders) as there are different assets, $\{(h, a), a=1, \ldots, A\}$. This is reminiscent of the structure imposed upon agents by the cash-in-advance constraint in Lu$\operatorname{cas}(1990))$. Each team member is in charge of a single asset. We refer to the traders as team members since the ex-ante objective function is shared by all of them. In particular we abstract from strategic behaviour. Member $a$ of investor $h$ 's team (denoted by $(h, a)$ ) submits the schedule for asset $a$ to the auctioneer, incorporating the following line of reasoning:

"I am rational in the sense that I know the equilibrium price function mapping shocks to prices. Unfortunately, I can only observe the price of asset $a$, and I cannot communicate with any of the other members $(h, b)$ who observe $q_{b}$ and trade $\theta_{b}^{h}, b \neq a$. ut, given the structure of the economy, I can update my beliefs as to the market environment faced by my team members from any 
one price. The resulting price of asset $a$ at which my order will be executed contains that information, allowing me to refine my information as to the prices of all the other assets and the trades of my team members."

The investors' optimization problem has the same team flavour that was analyzed, albeit in a somewhat simpler setting, in Marschak and Radner(1971)), Radner(1986)) and Radner(1986)). Vayanos(1999)) studies an economy where various agents in a hierarchical company allocate the company wealth to different assets when different team members have different private information about the final payoff of assets. In contrast, our model assumes for simplicity that information about the final payoff distribution of assets is homogeneous within a company $h$, but that different members have different information as to the current trading environment, and therefore about the traded values of those assets. The abstract properties of the trading environment defined by the dual frictions has been analyzed in $\mathrm{Zi}$ grand(2001)). In this paper we specialize the economy in order to compute a closed form solution and to study its asset pricing implications.

The structure of the economy is presented in Section 2. Section 3 introduces the investor's optimization problem, and Section 4 deals with the rational expectations equilibrium. Section 5 studies the asset pricing implications of our twin market microstructure assumptions. Subsection 5.1 shows that markets may exhibit arbitrage opportunities at the rational expectations equilibrium and Subsection 5.2 investigates the degree of informational efficiency at the equilibrium. In Subsection 5.3 the asset prices are contrasted to the Arrow-Debreu asset prices. Welfare is analyzed in Section 6 and Section 7 concludes.

\section{The Economy}

As mentioned in the introduction, individuals face two layers of risk in this economy, first about the trading environment ("state of information") and then about the realization of endowments and asset payoffs ("state of nature"). At time zero they face uncertainty about the market participants' endowments and preferences, which translates into uncertainty about prices. The asset demand functions they submit are not required to be either market or limit orders, but they are required to only depend on their own price, i.e. 
they need to be asset-specific. For instance, the demand schedule for asset $a$ is a function of the price of asset $a, q_{a}$, only, as opposed to the whole price vector $q$. At time one this uncertainty is resolved, asset orders get executed and the households consume. They are, by the very nature of immediacy risk, unable to retrade at the equilibrium price. The state of nature is realized at time two and final consumption occurs. Investor $h$ 's utility maximization program can then naturally be restated in the following fashion. Given that asset specific demand functions have to be handed over to the auctioneer before the state of information is realized, every investor's demand function for some asset $a$ can be thought of as being represented by a team member $(h, a)$ (we use the terms "trader" and "desk" interchangeably) as having the same preferences as the investor, and with the restriction that the $A$ members cannot communicate, so that $(h, a)$ 's private information corresponds to the observation of the price of asset $a, q_{a}$.

Preferences and endowments of all agents $h=1, \ldots H$ are assumed to be common knowledge, and the uncertainty as to the trading environment (i.e. the "state of information") comes for simplicity from liquidity, or noise, traders, aggregated as investor $H+1 . \epsilon_{a}$ represents the aggregate noise trader demand, and can be viewed as a market order. It is important for the sequel to recognize, though, that nothing in this model hinges on noise traders, they are solely chosen for simplicity. All that is really needed is that there be some uncertainty about the trading environment, for instance as to the preferences and endowments of all other traders, which is hardly a strong assumption. This is shown in Zigrand(2001)). In particular, we do not need noise trades in order to insure an imperfectly revealing rational expectations equilibrium, and noise trading is not driving our asset pricing results, such as the existence of arbitrage, informational inefficiencies or overreactions.

\section{The Investors' Decision Problem}

The simplifying assumptions which will guarantee a tractable problem are the following, denoted by $\mathbf{H}$.

H1 There is uncertainty at time zero as to the noise trades $\epsilon \in \mathcal{E}=\mathbb{R}^{A}$. We assume that the probability distribution $\mu$ of $\epsilon$ is multivariate normal with mean 0 and positive definite covariance matrix $\Sigma_{\epsilon}$, the $(a, b)$ th element of which is denoted by $\sigma_{a b}$. 
There is also uncertainty at time two as to the state of nature which represents the realization of the endowment and payoff random variables. We assume that the probability space of states of nature, $(S, p)$, is finite. $p_{s}>0$ represents the probability of state of nature $s$ occurring, and is common knowledge. Expectations with respect to $p$ are denoted by a superscript $p$ in order to distinguish them from the expectations with respect to $\mu$ which we write without a superscript.

H2 Asset payoffs are summarized by the $S \times A$ payoff matrix $R$, assumed to be of full rank (no redundant assets) and assumed to allow the existence of some asset with positive payoffs: $\exists y \in \mathbb{R}^{A}: R y>0$. The payoff of asset $a$ in state $s \geq 1$, equivalently the $(s, a)$ th element of $R$, is denoted by $d_{a, s}$.

Also, assume that $\sum_{b} E^{p}\left[d_{a} d_{b}\right] \sigma_{a b} \neq 0$, all $a \in A$. This is a regularity condition imposed upon parameters and is generically satisfied.

H3 Utility functions are quadratic quasi-linear: $u_{0}^{h^{\prime}}(x)=1$ and $u_{s}^{h^{\prime}}(x)=$ $c^{h}-d^{h} x, s=1, \ldots S$.

Investor $h$ owns initial endowments in each date and state $\omega_{s}^{h}, s=$ $0, \ldots S$. All endowments are common knowledge.

Assumption H3 satisfies a purpose similar to the constant absolute riskaversion assumption in standard rational expectations (REE) models in that it insulates asset demands from income effects. Also notice that the quasilinear utility functions have the additional characteristic that investors are risk-neutral with respect to time one consumption. Denote the equilibrium price function by $\phi: \mathcal{E} \rightarrow \mathbb{R}^{A}$, so that $q=\phi(\epsilon)$ is the equilibrium price vector when the noise traders supply $\epsilon$. By rational expectations, this function coincides with the investors' forecast function, as defined for instance in Lucas(1972)) or Radner(1979)).

The team member $(h, b)$ 's task consists in choosing an optimal trade in asset $b, \theta_{b}^{h}$, given that he can infer some information about $q_{-b}$ and about the quantity traded by all other team members, $\theta_{-b}^{h}=f_{-b}^{h}\left(q_{-b}\right)$, from his observation of $q_{b}$ : 


$$
\begin{array}{rl}
\max _{\theta_{b}^{h}} & E\left[U^{h} \| q_{b}\right] \\
= & \max _{\theta_{b}^{h}} E\left[u_{0}^{h}\left(\omega_{0}^{h}-\sum_{a \neq b} f_{a}^{h}\left(\phi_{a}\right) \phi_{a}-\theta_{b}^{h} q_{b}\right)\right. \\
& \left.+\sum_{s} p_{s} u_{s}^{h}\left(\omega_{s}^{h}+\sum_{a \neq b} d_{a, s} f_{a}^{h}\left(\phi_{a}\right)+d_{b, s} \theta_{b}^{h}\right) \| q_{b}\right]
\end{array}
$$

Then the first order condition with respect to $\theta_{b}^{h}$ for an interior solution is

$$
q_{b}=\sum_{s} d_{b, s}\left[\frac{E\left[p_{s} u_{s}^{h^{\prime}}\left(x_{s}^{h}\right) \| q_{b}\right]}{E\left[u_{0}^{h^{\prime}}\left(x_{0}^{h}\right) \| q_{b}\right]}\right]
$$

The term in the outer brackets, call it $\lambda_{s}^{h, b}$, is a state-price that is specific to asset $b$, or maybe to a specific market-maker or exchange. $\lambda_{s}^{h, b}$ cannot play the role of a stochastic pricing kernel since the $\lambda^{h, b}$ used in the pricing of asset $b$ does not typically price any other asset. And neither can the intertemporal marginal rate of substitution $\frac{p_{s} u_{s}^{h^{\prime}}\left(x_{s}^{h}\right)}{u_{0}^{h^{\prime}}\left(x_{0}^{h}\right)}$, whereas it can in standard models, again because it is not measurable with respect to the traders' information sets. This is the crucial asset-pricing implication of this model that will allow us to generate inefficient and mispriced equilibrium prices without recourse to ad-hoc assumptions. Informational innovations in one part of the markets are not immediately and simultaneously incorporated into all other prices, as standard REE models assume, because the decentralized trading structure prevents the various trading desks from instantaneously sharing the information extracted from prices. The trading orders of desk $b \in A$ incorporate desk $b$ 's estimates of the value of all open positions of assets other than $b$, as can be seen from the Euler equation above. 
Now using the fact that preferences are quasi-linear quadratic, the FOC of the household's optimization problem becomes:

$$
\begin{aligned}
q_{b} & =\sum_{s} p_{s} d_{b, s} E\left[c^{h}-d^{h}\left(\omega_{s}^{h}+\sum_{a} f_{a}^{h}\left(q_{a}\right) d_{a, s}\right) \| q_{b}\right] \\
& =\sum_{s} p_{s} d_{b, s}\left[c^{h}-d^{h} \omega_{s}^{h}-d^{h} \sum_{a} d_{a, s} E\left[f_{a}^{h}\left(q_{a}\right) \| q_{b}\right]\right] \\
& =\hat{q}_{b}^{h}-d^{h} \sum_{a} E^{p}\left[d_{a, s} d_{b, s}\right] E\left[f_{a}^{h}\left(q_{a}\right) \| q_{b}\right]
\end{aligned}
$$

where

$$
\hat{q}_{b}^{h} \equiv c^{h} E^{p}\left[d_{b, s}\right]-d^{h} E^{p}\left[d_{b, s} \omega_{s}^{h}\right]
$$

The expression $\hat{q}^{h}$ basically represents investor $h$ 's private valuation of the assets in an economy without noise. Notice that the demand function for asset $b$ depends on the demand functions of all other assets. Indeed, demand functions are the solution to a fixed-point problem, where each trader is forecasting the forecasts of the remaining team members.

In order to solve the problem, we need to compute the conditional expectation on the right-hand-side. Each team member knows that noise is normally distributed. Assume also that each investor has a linear forecast function $\phi: \phi(\epsilon)=\hat{F}+\hat{G} \epsilon$, where $\hat{F}$ is an $A \times 1$ vector of constants and $\hat{G}$ is an $A \times A$ matrix of constants. Given that $\epsilon \sim N\left(0, \Sigma_{\epsilon}\right)$, this induces a distribution of prices of $N\left(\hat{\mu}_{q}, \hat{\Sigma}_{q}\right)$, with $\hat{\mu}_{q}=\hat{F}$ and $\hat{\Sigma}_{q}=\hat{G} \Sigma_{\epsilon} \hat{G}^{\prime}$. The conditional expectations functions $E\left[q_{-a} \| q_{a}\right]$ are linear as well. Using these expressions, team member $(h, a)$ can easily deduce that $E\left[q_{a} \| q_{b}\right]=\nu_{a b}+v_{a b} \cdot q_{b}$, with $\nu_{a b}=\hat{\mu}_{q_{a}}-\hat{\mu}_{q_{b}} \frac{\hat{\sigma}_{q_{a}, q_{b}}}{\hat{\sigma}_{q_{b}}^{2}}$ and $v_{a b}=\frac{\hat{\sigma}_{q_{a}, q_{b}}}{\hat{\sigma}_{q_{b}}^{2}}$. The conjectured linearity is indeed preserved:

Proposition 1 (Demand Functions) For a linear forecast function, there are constants $\beta^{h} \in \mathbb{R}^{A}$ and $\alpha^{h} \in \mathbb{R}^{A}$ such that demand functions are of the form

$$
f_{a}^{h}\left(q_{a}\right)=\alpha_{a}^{h}-\beta_{a}^{h} q_{a}
$$

for all $a \in A$ and all $h \in H$.

The array of parameters $\beta^{h}$ summarizes three effects of prices on investor $h$ 's problem. Besides the traditional income (due to $\mathbf{H 3}$ there are actually 
none in this case) and substitution effects, it also comprises the information refinement effect via $\left\{v_{a b}\right\}_{a, b}$. As usual, $\mathbf{H 3}$ implies that in the absence of informational effects (i.e. $\hat{\Sigma}_{q}$ diagonal), demand is downward sloping, $\beta_{a}^{h}>0$, all $a$.

\section{Rational Expectations Equilibria}

We now solve for the linear REE of such an economy. For each given $\epsilon$, asset markets have to clear (commodity markets then clear by Walras' Law ${ }^{1}$ ). Denoting $\alpha_{a} \equiv \sum_{h} \alpha_{a}^{h}$, all $a \in A$, and similarly $\beta_{a} \equiv \sum_{h} \beta_{a}^{h}$, all $a \in A$,

$$
\sum_{h} f_{a}^{h}\left(q_{a}\right)=\alpha_{a}-\beta_{a} q_{a}=-\epsilon_{a} \quad ; \text { all } a \in A
$$

The solution is given by

$$
\phi(\epsilon)=\left[\begin{array}{c}
\vdots \\
\frac{\alpha_{a}}{\beta_{a}} \\
\vdots
\end{array}\right]+\operatorname{diag}\left(\cdots, \beta_{a}^{-1}, \cdots\right) \epsilon
$$

We now have to verify that indeed $\hat{F}=\left(\ldots, \frac{\alpha_{a}}{\beta_{a}}, \ldots\right)$ and that $\hat{G}=$ $\operatorname{diag}\left(\cdots, \beta_{a}^{-1}, \cdots\right)$, so that expectations are fulfilled. Define $\delta \equiv \frac{1}{\sum_{h}\left(d^{h}\right)^{-1}}$, $\delta^{h} \equiv \frac{\left(d^{h}\right)^{-1}}{\sum_{h}\left(d^{h}\right)^{-1}}$ and $\hat{q} \equiv \sum_{h} \delta^{h} \hat{q}^{h}$. Also define the operator diag as follows: if diag is applied to the vector $v \in \mathbb{R}^{n}$, it places the elements of $v$ on the diagonal of an $n \times n$ matrix of zeros, while if diag is applied to a square matrix, it generates the vector containing the diagonal elements. Finally denote the noncentral second moment matrix of asset payoffs, $R^{\prime} \operatorname{diag}(p) R$ by $M_{d}$, the $A \times A$ identity matrix by $I_{A}$ and the Hadamard (component-by-component) matrix product by $\circ$.

Proposition 2 (Existence) There always exists a unique (within the class of linear pricing functions) linear REE of the form

$$
\phi(\epsilon)=F+G \epsilon
$$


$F$ and $G$ are given by

$$
\begin{aligned}
& F=\hat{q} \equiv \sum_{h} \delta^{h} \hat{q}^{h} \\
& G=\delta I_{A} \circ\left[M_{d} \Sigma_{\epsilon}\left(I_{A} \circ \Sigma_{\epsilon}\right)^{-1}\right]
\end{aligned}
$$

By $\mathbf{H 2}, G$ is non-singular, i.e. $G$ is diagonal with typical element $G_{a a}=$ $\delta \sum_{b} E^{p}\left[d_{a} d_{b}\right] \frac{\sigma_{a b}}{\sigma_{a}^{2}} \neq 0$

The intercept $F$, the price vector in the absence of any noise, is the weighted average of the investors' private valuations, $\hat{q} \equiv \sum_{h} \delta^{h} \hat{q}^{h}$. It is also equal to the average price, $F=E[\phi]$ as well as to the standard CAPM valuation for an economy with assets that are in zero net supply.

We can in turn use this result to characterize the demand functions $f^{h}(q)=\alpha^{h}-\operatorname{diag}\left(\beta^{h}\right) q$ in more detail. Whether demand (whose slope is the inverse of the negative of $G$ ) is upward or downward sloping depends entirely on the exogenous parameters composing the matrices $M_{d}$ and $\Sigma_{\epsilon}$. From there it is clear that a negative slope is the norm, but not a necessity.

Investors trade among themselves and with noise traders. Since we can show from the proof of Proposition 1 that $\beta^{h}=\delta^{h} \cdot \beta$, each additional unit of liquidity supply induces a change of prices such that investor $h$ absorbs a fraction $\delta^{h}$ of that supply:

Lemma 1 At a REE, investor h's asset holdings can be split into a portfolio of assets that he trades with other investors and a portfolio holding a fraction $\delta^{h}$ of the noisy supply $\epsilon$ :

$$
\theta^{h}=\delta^{-1} \delta^{h} M_{d}^{-1}\left[\hat{q}^{h}-\hat{q}\right]-\delta^{h} \epsilon
$$

In what follows, it is useful to contrast our findings to the outcomes of the Radner equilibrium, by which we mean the competitive Arrow-Debreu equilibrium under uncertainty, but allowing for incomplete markets. In such a setting, investors maximize

$$
\left(\omega_{0}^{h}-\sum_{a} \theta_{a}^{h} q_{a}\right)+\sum_{s} p_{s}\left[c^{h}\left(\omega_{s}^{h}+\sum_{a} \theta_{a}^{h} d_{a s}\right)-\frac{1}{2} d^{h}\left(\omega_{s}^{h}+\sum_{a} \theta_{a}^{h} d_{a s}\right)^{2}\right]
$$

Asset demands are given by

$$
\theta^{h}=\delta^{-1} \delta^{h} M_{d}^{-1}\left(\hat{q}^{h}-q\right)
$$


Since the only active effect is the substitution effect, demands are downward sloping. ${ }^{2}$ Markets clear at an equilibrium, $\sum_{h=1}^{H} \theta^{h}+\epsilon=0$, which occurs if prices satisfy

$$
q^{R}=\phi^{R}(\epsilon)=\hat{q}+\delta M_{d} \epsilon
$$

\section{Asset Pricing}

In this section we analyze to what degree the microstructure generates asset prices that satisfy the list of pricing properties outlined in the introduction.

\subsection{Arbitrage}

The first effect that one may expect is that assets could be mispriced if investors faced the twin marketstructure assumptions. It is well-known that in standard economies the image of $\phi$ is a subset of $\mathcal{Q}$, the set of no-arbitrage prices defined, for our purposes, as follows:

Definition 1 A price vector $q$ admits No Arbitrage (NA) if the following holds:

$$
R y>0 \Rightarrow q^{\prime} y>0
$$

The set of such vectors is denoted by $\mathcal{Q}$, an open cone.

The realization $q$ admits no arbitrage if at that price any portfolio with a positive non-zero payoff has a positive cost. We show that the assumptions of immediacy risk and of asset-specificity (in conjunction with $\mathbf{H}$ ) indeed generate arbitrage opportunities with positive probability:

Proposition 3 Equilibria exhibit arbitrage opportunities with strictly positive probability.

This can be shown easily. Since $q=\phi(\epsilon)=F+G \epsilon$, with normal noise trades the range of possible prices is $\phi(\mathcal{E})=\mathbb{R}^{A}$, and unless all possible prices are no-arbitrage prices (which is ruled out by assumption $\mathbf{H 2}$ that guarantees that there be some asset $y$ paying off in at least one state, $R y>0$ ), the following inclusion is strict: $\mathcal{Q} \subset \phi(\mathcal{E})$. But then the event of price realizations $\phi(\mathcal{E}) \backslash \mathcal{Q}$ has strictly positive probability (both $\phi(\mathcal{E})$ and $\mathcal{Q}$ are open in $\mathbb{R}^{A}$, and $\mu$ is absolutely continuous with respect to $A$-dimensional Lebesgue measure) and consists entirely of arbitrages. ${ }^{3}$ 
Unfortunately, the assumptions required to derive a closed-form solution, in particular normality and quadratic quasi-linear preferences, make Proposition 3 look weaker than it actually is. The problem lies, of course, in the satiation and no-free disposal assumptions implicit in quadratic preferences. Whereas with standard non-satiated preferences, the Radner equilibrium does not allow for arbitrage, the same is not true for quadratic preferences if the support of $\epsilon$ is large enough. It can indeed be shown (see Zigrand(2001))) in a general model with non-satiated preferences that arbitrage is possible in our framework whereas it is impossible in the Radner economy. Here we will only be able to show that there are arbitrage opportunities that arise due to the information asymmetries that the microstructure imposes upon investors, which do not arise in the Radner economy.

As an example, consider the economy with the payoff matrix $\left[\begin{array}{ll}1 & 1 \\ 0 & 1\end{array}\right]$. The set of no-arbitrage prices is the set of prices for which $q_{2}>q_{1}>0$. Graphically:

\section{Insert figure 1 here}

Over and above the arbitrage opportunities that can survive at an equilibrium due to satiation and no-free disposal, there are more subtle arbitrage opportunities that arise in our framework that do not occur in the Radner economy. Since members cannot perfectly coordinate their asset purchases, the trading structure does not allow for complete risk-sharing. This is reflected in the fact that the marginal rates of substitution (MRS) of the different team members of the same investing entity $h$ differ, as $\lambda^{h, a}$ need not coincide with $\lambda^{h, b}, a \neq b$, because they are chosen based on different information. This is over and above the fact that the MRS between different investors may differ in general, even in the absence of the twin microstructure assumptions, if markets are incomplete $(A<S)$. The dislocation of the marginal rates of substitution is preserved at the equilibrium because the plausible trading structure introduced in this paper prevents perfect arbitraging, even if markets are complete. Consider for instance the arbitrage realization $q=(2,1)$. The reason investor $h$ is unable to make a riskless gain is that such an operation requires selling asset 1 and buying asset 2 . But neither trader 1 nor trader 2 knows whether $q_{2}<q_{1}$. But investors still try to benefit from what in effect amounts to a very good ex-ante deal, thereby bringing the MRS as close as possible given the segregated information structure. In the example above, trader 1 may for instance know that, for the given equilibrium probability distribution of prices, $q_{2}<q_{1}$ may be quite likely, prompting a 
short position in asset 1 . Trader 2 may or may not, again depending on the equilibrium distribution, go long in asset 2 . But even if indeed 1 goes short and 2 goes long, they do not know that they are engaging in an arbitrage scheme, and their portfolio is bounded, even when consumptions are below their satiation levels, or with nonsatiated preferences.

\subsection{Informational Efficiency}

Having shown that equilibria exist in the economy with immediacy risk, and having studied their allocational inefficiency, we shall analyze the information transmission across exchanges or across pits at these equilibria. If each pit is hit by an independent demand or supply shock and if no investors can trade on more than one pit, no information can be transmitted. In our setup, however, noises, and therefore equilibrium prices, are correlated so that each observed asset price reveals information about all the sources of noise. Still, markets generically remain informationally inefficient for any investor (in addition to being allocationally inefficient), in the sense that the investors, after having updated their information, possess less information than in the Radner economy:

Proposition 4 (Degree of Information Revelation to Traders) The dimension of residual uncertainty facing team member $(h, b)$ is $A-1$ with probability one.

A proof of a more general version of this proposition can be found in Zigrand(2001)), but this present case is particularly intuitive: since team member $(h, b)$ observes $q_{b}$ and knows that $q_{b}=\phi_{b}(\epsilon)=F_{b}-G_{b b} \epsilon_{b}$, he effectively knows $\epsilon_{b}$ (since $G_{b b} \neq 0$ ), and he can update his prior probabilities of $q_{-b}$ and $\epsilon_{-b}$ accordingly. But because $\Sigma_{\epsilon}$ is of full rank, all $A$ noise trades are only imperfectly correlated and $(h, b)$ is left with a finer posterior information set, but still with uncertainty about $A-1$ variables whose posterior support equals $\mathbb{R}$

meri c an 
In particular, the equilibrium is incompatible with an unrestricted (or even representative agent) economy since the information of the team as a whole is not compatible with Bayesian updating. An empirical analysis indicating a violation of Bayesian updating may therefore suggest irrational behaviour, even though agents are in fact very rational in trying to overcome the existing microstructure frictions. Relatedly, unlike in standard asset-pricing theory, the posterior probability assessments of the traders of the different assets, within the same team and across teams, are not equivalent, in the sense that the traders disagree on events of probability zero.

\subsection{Comparison to Radner Equilibria}

It seems natural to contrast the equilibrium just derived to the Radner equilibrium. For asset $a$, contrast the equilibrium price in the Radner model [R] (from Equation (2)) and in the immediacy-risk model [IR] (from Equation $(1))$ :

$$
\begin{aligned}
q_{a}^{R} & =\hat{q}_{a}+\delta \sum_{b} E^{p}\left[d_{a} d_{b}\right] \epsilon_{b} \\
q_{a} & =\hat{q}_{a}+\delta \sum_{b} E^{p}\left[d_{a} d_{b}\right] \frac{\sigma_{a b}}{\sigma_{a}^{2}} \epsilon_{a}
\end{aligned}
$$

We notice that in the expression for the equilibrium price for asset $a$ in the presence of immediacy risk, the conditional expectations function $E\left[\epsilon_{b} \| \epsilon_{a}\right]$ $=\frac{\sigma_{a b}}{\sigma_{a}^{2}} \epsilon_{a}$ replaces $\epsilon_{b}$. This is reminiscent of the certainty-equivalence results in quadratic decision problems, such as in Simon(1956)), but here it also applies to equilibrium variables.

Both equilibrium pricing functions coincide if $\epsilon_{a}=\ell_{a}\left(\epsilon_{1}\right)$, all $a$, where each $\ell_{a}$ is a Borel-measurable bijection, because the price of any single asset is fully revealing in that case. Assumption H1, by requiring that $\Sigma_{\epsilon}$ be positive definite, eliminates this possibility however. Alternatively, they coincide if $M_{d}$ is diagonal. Besides these two cases, assets may be under- or overpriced compared to $q^{R}$, their "fundamental value." But there is no regularity in the mispricing vis-à-vis the Radner value: $E\left[q_{a}-q_{a}^{R}\right]=E\left[q_{a}-q_{a}^{R} \| q_{a}\right]=0$, all $a$. The next proposition compares the volatilities:

Proposition 5 Assume that $M_{d}$ is not diagonal, and that assumption $\mathbf{H}$ holds. Then asset prices are less volatile than in the Radner equilibrium. 
There are two explanations for this. First, and by way of proof, conditional expectation functions are contractions, meaning they act as smoothers: since $q_{a}=E\left[q_{a}^{R} \| \epsilon_{a}\right]$ it follows that $\operatorname{var}\left(q_{a}^{R}\right) \geq \operatorname{var}\left(q_{a}\right)$. Second, the peculiarity of quasi-linear utility functions, whose indifference curves are not strictly contained in the positive orthant, contributes towards less volatility.

Our static model is clearly too stylized to capture many facets of realworld asset pricing. Nevertheless Proposition 4 and equations [R] and [IR] raise a very simple but important further point, namely as to where the often imperfect and noisy movements and comovements of the various assets come from. It has been repeatedly pointed out in the literature that there is considerable evidence that asset prices do not coincide with their "fundamental values." In particular refer for instance to the, mostly behavioural, literature on overreaction (for instance De Bondt and Thaler(1986)), Chopra, Lakonishok and Ritter(1992)) and Hong and Stein(1999))). Some empirical papers (see Summers(1986)) ) also argue that there are limits to arbitrage which prevent the wedge between prices and fundamental values (however defined) from closing. De Long, Shleifer, Summers and Waldmann(1990)), Dow and Gorton(1994)) and Shleifer and Vishny(1997)) propose various models to address that point.

In a Walrasian world the global and omniscient auctioneer impounds all relevant information into all prices when forcing a global market clearing, and (refer to equation $[\mathrm{R}]$ ) all the various factors, no matter how unrelated, are impounded immediately and correctly into prices. But given that in reality there is no such auctioneer, that investors do not submit global Marshallian demand schedules, and that markets tend to be segmented, if only according to the two frictions introduced in this present paper, the Walrasian answer can at best be an approximation. The failure of markets to follow such perfect pricing, however, should not be taken as proof that investors necessarily behave in an irrational fashion, such as succumbing to over- or underreactions. In conjunction with other, possibly behavioural factors, one of the contributing determinants to the aforementioned empirical regularities and stylized facts may be the twin microstructure assumption in our framework:

Proposition 6 Compared to the standard model, asset prices may appear to over-or underreact to contemporaneous news when compared to the Radner benchmark:

$$
\frac{\partial q_{a}}{\partial \epsilon_{a}}>\frac{\partial q_{a}^{R}}{\partial \epsilon_{a}}>0 \quad \text { or } \quad \frac{\partial q_{a}}{\partial \epsilon_{a}}<\frac{\partial q_{a}^{R}}{\partial \epsilon_{a}}
$$


Overreaction is more likely than underreaction.

Overreaction occurs when $\frac{\partial q_{a}}{\partial \epsilon_{a}}>\frac{\partial q_{a}^{R}}{\partial \epsilon_{a}}$, i.e. when $\delta \sum_{b} E^{p}\left[d_{a} d_{b}\right] \frac{\sigma_{a b}}{\sigma_{a}^{2}}>$ $\delta E^{p}\left[d_{a}^{2}\right]$, i.e. when $\sum_{b \neq a} E^{p}\left[d_{a} d_{b}\right] \sigma_{a b}>0$. Upon inspection, it is intuitive that a positive sign is more likely. Assume for simplicity that $A=2$. All that is needed for overreaction is that $E^{p}\left[d_{1} d_{2}\right]$ and $\sigma_{12}$ have the same sign. It is plausible that when the payoffs of assets $a$ and $b$ are positively related, so are the liquidity trades. Since in our model liquidity traders represent the initial uncertainty about the traders' preferences and wealth, when actually modelled they would hold a diversified portfolio. So if a need for liquidation arises, the liquidity traders will liquidate similar assets concurrently, and conversely if they go long a diversified portfolio. If that is the case, trader $(h, a)$ compensates his lack of contemporaneous information about all other assets by overly relying on the information contained in his own piece of information. To conclude, overreactions seem more plausible than underreactions.

Furthermore, it is apparent that the size of any overreaction is proportional to $\delta$ which, together with consumption, determines aggregate absolute risk-aversion. Ceteris paribus, this leads us to the intuitive idea that serious over- or underreactions are more likely to occur in environments characterized by high effective risk-aversion, such as in periods of crises. Recent stock market evidence seems to unambiguously support the idea that effective risk-aversion goes up in periods of crisis (refer for instance to the model in Danielsson and Zigrand(2001)) where the current regulatory environment is responsible for this pattern). This effect is even reinforced in recent stock market history where effectively the correlation between returns of different assets has risen in periods of uncertainty and crises (refer for instance to Danielsson, Shin and Zigrand(2001)) for a model), creating even more pronounced overreactions, as predicted by Proposition 6 .

\section{Welfare}

In order to compare the ex-ante utility of investor $h$ across the two trading arrangements, we first compute the equilibrium asset demands in both cases.

In the frictional economy, we know from Lemma 1 that:

$$
\theta^{h}=\delta^{-1} \delta^{h} M_{d}^{-1}\left[\hat{q}^{h}-\hat{q}\right]-\delta^{h} \epsilon
$$


which turns out to be exactly equivalent to the Radner equilibrium asset allocation. Intuitively, quadratic quasi-linear preferences aggregate in such a way that each investor $h$ holds a fraction $\delta^{h}$ of the noisy supply, the effective market portfolio. It follows that at a Radner equilibrium each investor's demand for asset $a$ only depends on the noise trades of asset $a, \epsilon_{a}$, exactly as in the frictional economy where such a dependence is imposed by the nature of trades. In particular, if the Radner economy displayed mean-variance efficient portfolio allocations, the same would be true in the immediacyrisk economy, despite the serious information processing constraints imposed upon traders by the environment. We can extend these findings:

Proposition 7 Ex-ante welfare and equilibrium asset allocations are identical to the ones in the Radner economy. If furthermore $M_{d}$ is diagonal, then consumption allocations are identical as well, in all states and dates, $\epsilon$ a.s.

This proposition cautions against inferring welfare conclusions from asset prices. Here the behaviour of asset prices is quite distinct from the behaviour they would have in a first-best world, but welfare is nevertheless the same. It is the rationality of agents together with market clearing that are able, in some sense, to indirectly overcome some of the impediments to trade. This is reminiscent of the results found by Constantinides(1986)), albeit in a different context: demand functions may be vastly different from the frictionless ones, but they allow the agents to reach a level of satisfaction remarkably close to the level they enjoyed in the perfect benchmark economy.

\section{Conclusion}

We show how a plausible market microstructure could display realistic patterns usually attributed to irrational valuation, such as existence of arbitrage, informational inefficiencies, overreactions and so forth.

The model we construct is very simple, though, for we want to compute the REE in closed-form. In that respect we believe that a dynamic continuous-time extension may be useful to capture explicitly how rational market prices may stray from their fundamental values, as well as from the prices of their replicating portfolios, for a period time.

If reasonably profitable arbitrage opportunities could be sustained, a further extension may be to allow agents, upon investing an amount $c>0$ in the necessary technology, to have instantaneous access to the floors and 
perfect communication, and therefore to trade without immediacy risk, as in Zigrand(1999)). The number of such (strategic) arbitrageurs is endogenous. The interesting questions concern the degree of information revealed via the arbitrageurs' cross-pit trades, especially as $c \rightarrow 0$, for now team member $(h, a)$ can also take into consideration when updating that $q_{a}$ reveals something about the arbitrageurs' trades, including about their trades in assets other than $a$. This would illustrate the belief by practitioners that arbitrageurs improve upon the allocational and informational efficiency of prices. 


\section{A Proofs}

Proof of Proposition 1. To compute the demands of a rational agent, we conjecture that $f_{a}^{h}\left(q_{a}\right)$ is an affine function for all $a: f_{a}^{h}\left(q_{a}\right)=\alpha_{a}^{h}-\beta_{a}^{h} q_{a}$. Rewriting the asset demand function accordingly and using $E\left[q_{a} \| q_{b}\right]=\nu_{a b}+$ $v_{a b} q_{b}$ yields

$$
q_{b}=\hat{q}_{b}^{h}-d^{h} \sum_{a} E^{p}\left[d_{a, s} d_{b, s}\right]\left[\alpha_{a}^{h}-\beta_{a}^{h}\left\{\nu_{a b}+v_{a b} q_{q}\right\}\right]
$$

which generates the system of $2 A$ equations in $2 A$ unknowns

$$
\begin{aligned}
d^{h} \sum_{a} E^{p}\left[d_{a, s} d_{b, s}\right] \beta_{a}^{h} v_{a b} & =1 \quad(\forall b \in A) \\
d^{h} \sum_{a} E^{p}\left[d_{a, s} d_{b, s}\right] \alpha_{a}^{h} & =\hat{q}_{b}^{h}+d^{h} \sum_{a} E^{p}\left[d_{a, s} d_{b, s}\right] \beta_{a}^{h} \nu_{a b} \quad(\forall b \in A)
\end{aligned}
$$

We define the matrices

$$
\begin{aligned}
B^{h} & \equiv d^{h} M_{d} \\
W^{h} & \equiv\left[\begin{array}{ccc}
\ddots & & \\
& \hat{\sigma}_{q_{a}^{2}}^{2} & \\
& & \ddots
\end{array}\right] \hat{\Sigma}_{q} \circ B^{h}
\end{aligned}
$$

The block diagonal system can be written as

$$
\begin{aligned}
W^{h} \beta^{h} & =\mathbf{1} \\
B^{h} \alpha^{h} & =\hat{q}^{h}+\left(B^{h}\left(\operatorname{diag}\left(\beta^{h}\right)\right)-I\right) \hat{F}
\end{aligned}
$$

Only the second equality warrants elaboration:

$$
\begin{aligned}
B^{h} \alpha^{h} & =\hat{q}^{h}+\left\{\left(\mathbf{1} \hat{\mu}_{q}^{\prime}\right) \circ B^{h}-\left(\operatorname{diag} \hat{\mu}_{q}\right) W^{h}\right\} \beta^{h} \\
& =\hat{q}^{h}+\left(\left(\mathbf{1} \hat{\mu}_{q}^{\prime}\right) \circ B^{h}\right) \beta^{h}-\left(\operatorname{diag} \hat{\mu}_{q}\right) \mathbf{1} \\
& =\hat{q}^{h}+\left\{\left(\left(\mathbf{1 1}^{\prime}\right)\left(\operatorname{diag} \hat{\mu}_{q}\right)\right) \circ B^{h}\right\} \beta^{h}-\left(\operatorname{diag} \hat{\mu}_{q}\right) \mathbf{1} \\
& =\hat{q}^{h}+\left\{B^{h} \circ\left(\left(\mathbf{1 1}^{\prime}\right)\left(\operatorname{diag} \hat{\mu}_{q}\right)\right)\right\} \beta^{h}-\left(\operatorname{diag} \hat{\mu}_{q}\right) \mathbf{1} \\
& =\hat{q}^{h}+\left\{\left(B^{h} \circ\left(\mathbf{1 1}^{\prime}\right)\right)\left(\operatorname{diag} \hat{\mu}_{q}\right)\right\} \beta^{h}-\left(\operatorname{diag} \hat{\mu}_{q}\right) \mathbf{1} \\
& =\hat{q}^{h}+B^{h}\left(\operatorname{diag} \hat{\mu}_{q}\right) \beta^{h}-\left(\operatorname{diag} \hat{\mu}_{q}\right) \mathbf{1} \\
& =\hat{q}^{h}+B^{h}\left(\operatorname{diag} \beta^{h}\right) \hat{\mu}_{q}-\hat{\mu}_{q}
\end{aligned}
$$


We used the fact that $\mathbf{A} \circ(\mathbf{B C})=(\mathbf{A} \circ \mathbf{B}) \mathbf{C}$ if $\mathbf{C}$ is diagonal. This completes the proof of the second equality.

Finally, we show that within the class of linear demands, the vectors of constants $\alpha^{h}$ and $\beta^{h}$ are defined uniquely, namely that the matrices $B^{h}$ and $W^{h}$ are nonsingular. Recall that if $\mathbf{A}$ is a diagonal matrix, then $(\mathbf{A B}) \circ \mathbf{C}=$ $\mathbf{A}(\mathbf{B} \circ \mathbf{C})$. By Schur's product theorem we know that $\hat{\Sigma}_{q} \circ B^{h}$ is positive definite. $W^{h}$ is therefore invertible as the product of two invertible matrices. This completes the proof of invertibility, and the proof of the proposition.

Finally, we prove the statement in the text that in the absence of informational effects, demands are downward sloping. Indeed, it is obvious that if $\mathbf{A}$ and $\mathbf{B}$ are both diagonal, then $\mathbf{A B}=\mathbf{A} \circ \mathbf{B}$. Hence $W_{a b}^{h}=0, a \neq b$, $\beta_{a}^{h^{-1}}=W_{a a}^{h}=\sigma_{\epsilon_{a}}^{2} d^{h} E^{p}\left[\left(d_{a, s}\right)^{2}\right]>0$.

Proof of Proposition 2 (Existence). We can solve recursively for $G$ and $F$. As far as $G$ is concerned, we saw that $G=\operatorname{diag}\left(\cdots, \beta_{a}^{-1}, \cdots\right)$. Hence at a REE

$$
G^{-1}=\delta^{-1} \operatorname{diag}\left[\left\{\left[\begin{array}{ccc}
\ddots & & \\
& \hat{\sigma}_{q a}^{-2} & \\
& & \ddots
\end{array}\right]\left(G \Sigma_{\epsilon} G\right) \circ M_{d}\right\}^{-1} \mathbf{1}\right]
$$

which is true iff

$$
\begin{gathered}
\operatorname{diag}\left(G^{-1}\right)=\delta^{-1}\left\{\left[\begin{array}{ccc}
\ddots & & \\
& \hat{\sigma}_{q_{a}}^{-2} & \\
0 & & \ddots
\end{array}\right]\left(G \Sigma_{\epsilon} G\right) \circ M_{d}\right\}^{-1} \mathbf{1} \\
\Leftrightarrow\left\{\left[\begin{array}{ccc}
\ddots & & 0 \\
& \hat{\sigma}_{q_{a}}^{-2} & \\
0 & & \ddots
\end{array}\right]\left(G \Sigma_{\epsilon} G\right) \circ M_{d}\right\} \operatorname{diag}\left(G^{-1}\right)=\delta^{-1} \mathbf{1} \\
\Leftrightarrow\left\{\frac{\sigma_{a b} G_{b b}}{\sigma_{a}^{2} G_{a a}} E\left[d_{a} d_{b}\right]\right\}_{(a, b)}\left[\begin{array}{c}
\vdots \\
G_{b b}^{-1} \\
\vdots
\end{array}\right]=\delta^{-1} \mathbf{1}
\end{gathered}
$$

from which the expression for $G_{a a}$ follows uniquely. The last derivation used the fact that the $(a, b)$ th element of the matrix $\left[\begin{array}{ccc}\ddots & & \\ & \hat{\sigma}_{q a}^{-2} & \\ & & \ddots \\ 0 & & \ddots\end{array}\right] G \Sigma_{\epsilon} G$ is $\frac{\sigma_{a b} G_{b b}}{\sigma_{a}^{2} G_{a a}}$, where $\sigma_{a b}$ is the $(a, b)$ th element of $\Sigma_{\epsilon}$. 
We now derive the intercept $F$. Recall that $F=\operatorname{diag}(\beta)^{-1} \alpha$. Now

$$
\begin{aligned}
\alpha & \equiv \sum_{h} \alpha^{h} \\
& =\sum_{h}\left(B^{h}\right)^{-1} \hat{q}^{h}+\sum_{h}\left(B^{h}\right)^{-1}\left(B^{h} \operatorname{diag}\left(\beta^{h}\right)-I\right) F \\
& =\sum_{h}\left(B^{h}\right)^{-1} \hat{q}^{h}+\sum_{h}\left(\operatorname{diag}\left(\beta^{h}\right)-\left(B^{h}\right)^{-1}\right) F \\
& =\sum_{h}\left(B^{h}\right)^{-1} \hat{q}^{h}+\left(\operatorname{diag}(\beta)-\sum_{h}\left(B^{h}\right)^{-1}\right) F
\end{aligned}
$$

It follows that

$$
\begin{aligned}
F= & \operatorname{diag}(\beta)^{-1} \alpha \\
= & \operatorname{diag}(\beta)^{-1} \sum_{h}\left(B^{h}\right)^{-1} \hat{q}^{h}+\left(I-\operatorname{diag}(\beta)^{-1} \sum_{h}\left(B^{h}\right)^{-1}\right) F \\
\Leftrightarrow 0= & \operatorname{diag}(\beta)^{-1} M_{d}^{-1}\left(\sum_{h}\left(d^{h}\right)^{-1} \hat{q}^{h}\right) \\
& -\operatorname{diag}(\beta)^{-1} \delta^{-1} M_{d}^{-1} F
\end{aligned}
$$

Proof of Lemma 1. We know that $\theta^{h}=\alpha^{h}-\operatorname{diag}\left(\beta^{h}\right) q$. From the proof of Proposition 1, $\mathbf{1}=W^{h} \beta^{h}=d^{h}\left(\left[\begin{array}{ccc}\ddots & & \\ & \hat{\sigma}_{q_{a}}^{-2} & \\ 0 & & \ddots\end{array}\right] \hat{\Sigma}_{q} \circ M_{d}\right) \beta^{h}$. Solving for $\beta^{h}$ we get

$$
\beta^{h}=\delta^{-1} \delta^{h}\left(\left[\begin{array}{ccc}
\ddots & & \\
& \hat{\sigma}_{q a}^{-2} & \\
0 & & \ddots
\end{array}\right] \hat{\Sigma}_{q} \circ M_{d}\right)^{-1} \mathbf{1}
$$

Summing across $h=1, \ldots H$, we find that $\beta^{h}=\beta \delta^{h}$.

Also from Proposition 1,

$$
\begin{aligned}
\alpha^{h} & =\left(d^{h}\right)^{-1} M_{d}^{-1}\left[\hat{q}^{h}+d^{h} \delta^{h} M_{d}(\operatorname{diag}(\beta)) \hat{q}-\hat{q}\right] \\
& =\delta^{-1} \delta^{h} M_{d}^{-1}\left(\hat{q}^{h}-\hat{q}\right)+\delta^{h}(\operatorname{diag}(\beta)) \hat{q}
\end{aligned}
$$


from which it follows that $\alpha=\sum_{h} \alpha^{h}=(\operatorname{diag}(\beta)) \hat{q}$.

Now since individual demand is $\theta^{h}=\alpha^{h}-\operatorname{diag}\left(\beta^{h}\right) q$, summing over $h$ we find that $\operatorname{diag}(\beta) q=\alpha+\epsilon$. Inserting this expression back into the individual demands, we get $\theta^{h}=\delta^{-1} \delta^{h} M_{d}^{-1}\left(\hat{q}^{h}-\hat{q}\right)-\delta^{h} \epsilon$.

Proof of Proposition 7. We saw that asset allocations agree in both economies, so that in particular consumptions $x_{s}^{h}, s \geq 1$ agree as well, for almost all $\epsilon$. Of course, we showed that period zero prices do not agree, and thus that period zero consumption possibly doesn't either. Still, we shall now argue that, due to the assumed quasi-linearity of preferences, the expected consumption, and thus the expected utility, is the same in both economies.

First have a look at the Radner economy, and recall again that $\theta^{h}=$ $\left(d^{h}\right)^{-1} M_{d}^{-1}\left[\hat{q}^{h}-\hat{q}\right]-\delta^{h} \epsilon$ and $q^{R}=\hat{q}+\delta M_{d} \epsilon$. It follows that

$$
\begin{aligned}
x_{0}^{R, h} & =\omega_{0}^{h}-\theta^{h} \cdot q^{R} \\
& =\omega_{0}^{h}-\delta^{h}\left[\delta^{-1}\left(\hat{q}^{h}-\hat{q}\right)^{\prime} M_{d}^{-1} \hat{q}+\left(\hat{q}^{h}-\hat{q}\right)^{\prime} \epsilon-\hat{q}^{\prime} \epsilon-\delta \epsilon^{\prime} M_{d} \epsilon\right]
\end{aligned}
$$

In the IR economy, on the other hand, investor $h$ consumes

$$
x_{0}^{h}=\omega_{0}^{h}-\delta^{h}\left[\delta^{-1}\left(\hat{q}^{h}-\hat{q}\right)^{\prime} M_{d}^{-1} \hat{q}+\delta^{-1}\left(\hat{q}^{h}-\hat{q}\right)^{\prime} M_{d}^{-1} G \epsilon-\hat{q}^{\prime} \epsilon-\epsilon^{\prime} G \epsilon\right]
$$

Unless $G=\delta M_{d}$, consumptions do not typically coincide. It is apparent that the consumptions do coincide $\epsilon$ a.s. if $M_{d}$ is diagonal, since then $M_{d}^{-1} G=M_{d}^{-1}\left(\left[M_{d} \Sigma_{\epsilon}\left(I_{A} \circ \Sigma_{\epsilon}\right)^{-1}\right] \circ \delta I_{A}\right)=M_{d}^{-1} M_{d}\left(\left[\Sigma_{\epsilon}\left(I_{A} \circ \Sigma_{\epsilon}\right)^{-1}\right] \circ \delta I_{A}\right)=$ $\left[\Sigma_{\epsilon}\left(I_{A} \circ \Sigma_{\epsilon}\right)^{-1}\right] \circ \delta I_{A}=\delta I_{A}$.

But as far as ex-ante welfare is concerned, all we need to show is that expected consumptions agree. Using the fact that $E[\epsilon]=0$, we need to establish that $\delta E\left[\epsilon^{\prime} M_{d} \epsilon\right]=E\left[\epsilon^{\prime} G \epsilon\right]$. Now $E\left[\epsilon^{\prime} M_{d} \epsilon\right]=\mathbf{1}^{\prime}\left[M_{d} \circ \Sigma_{\epsilon}\right] \mathbf{1}$, and $E\left[\epsilon^{\prime} G \epsilon\right]=\sum_{a} G_{a a} \sigma_{a a}=\sum_{a} \sigma_{a a} \delta \sum_{b} E^{p}\left[d_{a} d_{b}\right] \frac{\sigma_{a b}}{\sigma_{a a}}$ which indeed simplifies to the required expression. 


\section{References}

Chopra, Navin and Josef Lakonishok and Jay R. Ritter (1992). "Measuring Abnormal Performance. Do Stocks Overreact?" Journal of Financial Economics 31, pp. 235-268

Constantinides, George (1986). "Capital Market Equilibrium with Transaction Costs," Journal of Political Economy 94-4, pp. 842-862

Danielsson, Jón and Hyun Song Shin and Jean-Pierre Zigrand (2001). "Asset Price Dynamics with Value-at-Risk Constrained Traders," FMG Discussion Paper 394

Danielsson, Jón and Jean-Pierre Zigrand (2001). "What Happens when you Regulate Risk? Evidence from a Simple Equilibrium Model," FMG Discussion Paper 393

De Bondt, Werner and Richard H. Thaler (1986). "Does the Stock Market Overreact?" Journal of Finance 40-3, pp. 793-807

De Long, Bradford and Shleifer, Andrei and Summers, Lawrence and Robert Waldmann (1990), "Noise Trader Risk in Financial Markets," Journal of Political Economy 98-4, pp. 703-738

Dow, James and Gary Gorton (1994). "Arbitrage Chains," Journal of Finance 49-3, pp. 819-849

Hong, Harrison and Jeremy Stein (1999). "A Unified Theory of Underreaction, Momentum Trading, and Overreaction in Asset Markets," Journal of Finance 54, pp. 2143-2184

Kyle, Albert (1985). "Continuous Auctions and Insider Trading," Econometrica 53, pp. 1315-1336

Lucas, Robert (1972). "Expectations and the Neutrality of Money," Journal of Economic Theory 4, pp. 103-124

Lucas, Robert (1990). "Liquidity and Interest Rates," Journal of Economic Theory 50, pp. 237-264

Marschak, Jacob and Roy Radner (1971). The Economic Theory of Teams, Yale University Press

Radner, Roy (1979). "Rational Expectations Equilibrium: Generic Existence and the Information Revealed by Price," Econometrica 47, pp. $655-678$ 
Radner, Roy (1986). "Teams," in Decision and Organization. Essays in Honor of Jacob Marschak, C. B. McGuire and Roy Radner (eds), pp. 189-215, University of Minnesota Press

Radner, Roy (1986). "Allocation of a Scarce Resource under Uncertainty: An Example of a Team," in Decision and Organization. Essays in Honor of Jacob Marschak, C. B. McGuire and Roy Radner (eds), pp. 217-236, University of Minnesota Press

Shleifer, Andrei and Robert Vishny (1997). "The Limits of Arbitrage," Journal of Finance 52, pp. 35-55

Simon, Herbert (1956). "Dynamic Programming under Uncertainty with a Quadratic Objective Function," Econometrica 24, pp. 74-81

Summers, Lawrence (1986). "Does the Stock Market Rationally Reflect Fundamental Values?" Journal of Finance 41-3, pp. 591-601

Vayanos, Dimitrios (1999). "Optimal Decentralization of Information Processing in the Presence of Synergies," Mimeo MIT

Zigrand, Jean-Pierre (1999). "Arbitrage and Endogenous Market Integration," FMG Discussion Paper 319

Zigrand, Jean-Pierre (2001). "Rational Limits to Arbitrage," FMG Discussion Paper 392 


\section{Endnotes}

${ }^{1}$ Equilibrium requires that at all $s=0, \ldots, S, \sum_{h} x_{s}^{h}+x_{s}^{\epsilon}=\sum_{h} \omega_{s}^{h}+\omega_{s}^{\epsilon}$ (where $\omega_{s}^{\epsilon}$ is the noise traders' endowment in state $s$ ). At $s=0$, the budget constraints imply that $x_{0}^{h}=\omega_{0}^{h}-q \cdot f^{h}$ and $x_{0}^{\epsilon}=\omega_{0}^{\epsilon}-\epsilon \cdot q$. It is then easy to verify that these demands do indeed sum up to the aggregate endowment when asset markets clear. The same is true for $s \geq 1$.

${ }^{2} \mathrm{~A}$ (differentiable) demand function $f$ is said to be downward sloping at $q$ if its Jacobian is negative quasidefinite. Intuitively, this follows from the observation that by definition of negative quasidefiniteness $(d q)^{\prime} \partial f(q)(d q)<$ 0 for $d q \neq 0$, i.e. $(d q)^{\prime} d \theta<0$ : prices and quantities of portfolios move in opposite directions.

${ }^{3}$ Even though events in $\phi(\mathcal{E}) \backslash \mathcal{Q}$ look like an arbitrage to an econometrician or outside observer, they are not, however, arbitrages that the different traders in team $h$ could profit from since the arbitrage opportunity is not common knowledge to them. Section 5.2 elaborates upon this. In Zigrand(2001)) we show in a general framework that $\mathcal{Q} \subset \phi(\mathcal{E}) \subset \mathcal{Q}^{C K}$, where $\mathcal{Q}^{C K}$ is the set of price vectors for which it is not common knowledge to any subset of members of the same team that there is an arbitrage. The simplifying assumptions in this paper guarantee that $\mathcal{Q}^{C K}=\mathbb{R}^{A}$, allowing the existence of a closed-form solution. 


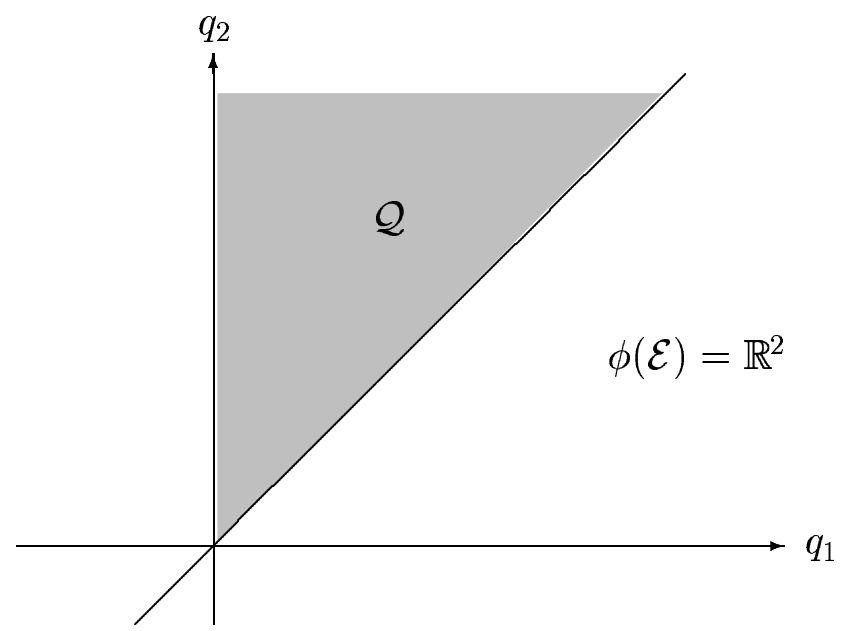

Figure 1: The SET of NO-ARBitRAGE PRICES, $\mathcal{Q}$, IS AN OPEN CONVEX CONE AND A STRICT SUBSET OF $\phi(\mathcal{E})=\mathbb{R}^{2}$. The PRICE REALIZATIONS IN THE UNSHADED AREA REPRESENT ARBITRAGE OPPORTUNITIES IN THE SUPPORT OF THE PRICE DISTRIBUTION. 